\title{
IMPACT OF RESISTIVE-WALL WAKE FIELDS GENERATED BY LOW- GAP CHAMBERS ON THE BEAM AT THE ESRF
}

\author{
R. Nagaoka, ESRF, Grenoble, France
}

\begin{abstract}
The observation and analysis of betatron tune shifts with current made at the ESRF are presented. The concerned tune shifts are supposed to arise from the mean quadrupolar wake field induced by the resistive low-gap chambers, which are distributed around the ring for insertion devices. By employing the scheme developed by K. Yokoya, fields are computed for the actual low-gap chambers of the ESRF machine. Effective focusing strengths are then deduced to compute the tune shifts of individual bunches in an arbitrary filling. It is found that the multi-turn effect plays a key role in reproducing the observed tune shift in multibunch fillings. Tune shifts are also expected to be significant in single bunch due to the strong short-range resistive-wall wake field. Experimental evidence of the numerical finding is pursued. The impact to the coherent single bunch tune shifts and hence to the modelling of the broadband impedance is also discussed
\end{abstract}

\section{INTRODUCTION}

Betatron tune shifts with current, with opposite signs for the horizontal and vertical, which become larger than two synchrotron-side bands at the operating current of $200 \mathrm{~mA}$, were for long a puzzle at the ESRF. The increase of the horizontal tune with current particularly made it difficult to relate this to an impedance effect (Fig. 1).
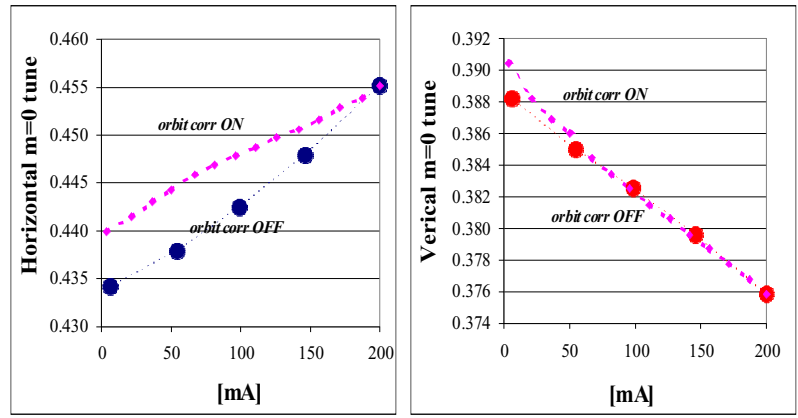

Figure 1: Measured current-dependent betatron tune shifts, with and without the orbit correction.

After confirming that, 1) the effect is not due to a currentdependent closed-orbit drift that may cause tune shifts, 2) tune shifts are nearly independent of the beam filling and depend only on the average current, the resistive-wall wake field was suspected owing to its long-range nature. In fact in literature, we find that resistive-wall chambers having an asymmetric cross section generate a quadrupolar mean field. Namely, in the absence of any coherent motion and having the beam centre on the machine axis, individual particles may feel the focusing (defocusing) force of the wake field [1][2]. Such tune shifts were also observed in PEP II. S. Heifets computed the tune shifts in terms of the wake field and found a reasonably good agreement with the measurement [3].

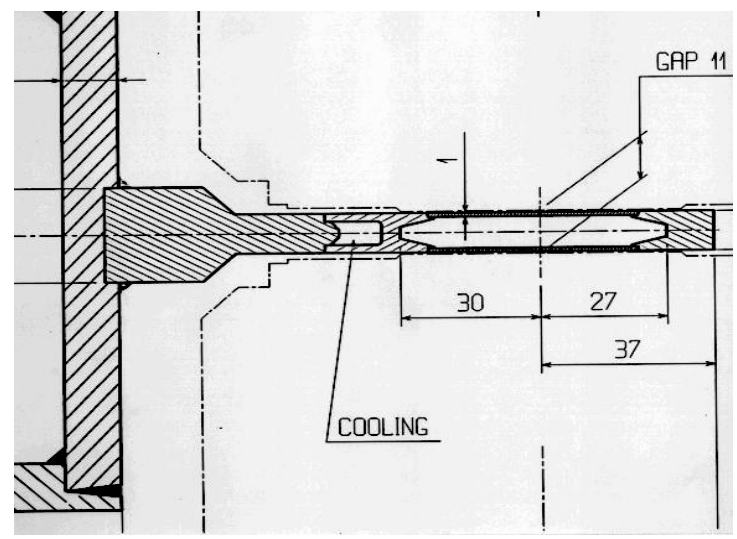

Figure 2: ESRF $10 \mathrm{~mm}$ (internal = $8 \mathrm{~mm}$ ) chamber.

The present work aims to make such analysis, above all to study the impact of resistive-wall wakes on the tune shift, in different filling modes. The work of K. Yokoya is used, which allows numerical computations of long and short-range fields, generated by a particle at any position in a resistive-wall chamber of an arbitrary cross section [2]. Wake fields are computed for the ESRF low-gap chambers (Fig. 2) and introduced according to the actual configuration of the chambers in the machine. The study of classical resistive-wall instabilities is out of the scope of this paper.

\section{WAKE FIELD COMPUTATION}

A code was developed to compute the transverse wake field according to the formulation of Ref. 2. The method seeks for a solution by dividing the field into a part driven by the source in a perfectly conducting wall, and another, due to a source-less resistive wall. The latter that obeys the Kirchhoff integral formula is solved in the frequency domain. The formalism takes well account of the fact that the wake force felt by the trailing (witness) particle depends explicitly on its own position when the chamber cross section has no circular symmetry. Thus it treats both the source and the witness particles in a general manner, which is applicable to all longitudinal ranges (short and long), in a way suited for the numerical computation of the field, for an arbitrary chamber cross section. The numerical procedure consists in discretising the chamber circumference into grid points and performing matrix manipulations of the fields evaluated at the grid points. 
The transverse wake fields were computed for two ESRF low-gap chambers, i.e. the 10 and $15 \mathrm{~mm}$ stainless steel chambers, which are found to give the major contribution. The internal vertical full apertures are respectively 8 and $11 \mathrm{~mm}$. As regards the $10 \mathrm{~mm}$ vessel, there is a $50 \mu \mathrm{m}$ copper coating on the vertical surface, which however was not taken into account in the present study, as the skin depth of the concerned field is expected to be much larger. The field of interest, namely, that felt by the trailing particle when the source particle is at the origin, is shown in Figs. 3 for the two chambers.
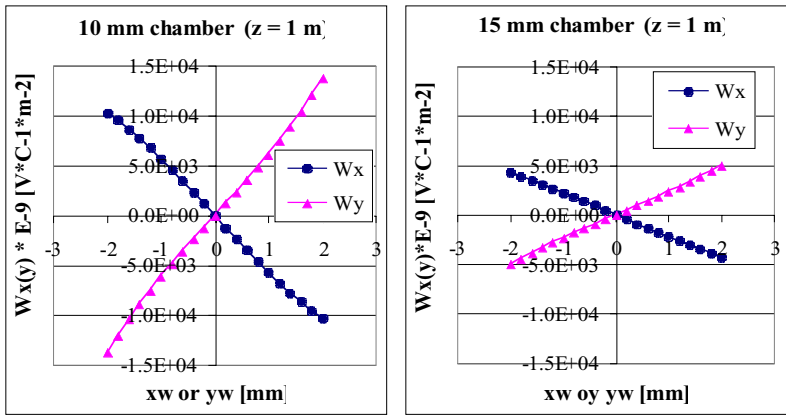

Figure 3: Calculated transverse wake fields for the 10 (left) and $15 \mathrm{~mm}$ (right) ESRF chambers.

\section{IMPACT ON MULTIBUNCH}

The incoherent tunes of the individual bunches were computed on the basis of the wake fields with an optics code developed for this purpose. The computation takes into account the low-gap chambers as how they are actually installed in the ring. The focusing strength of a low-gap chamber felt by a given bunch is computed by taking into account the beam filling structure as well as the multi-turn effect.
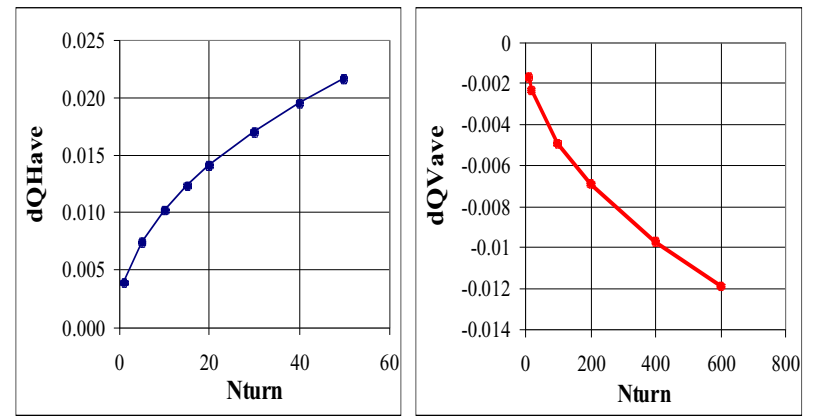

Figure 4: Calculated dependence of current-dependent betatron tune shift on multi-turns.

The results showed, first of all, that the single turn effect is much too small to explain the measured tune shifts, which appears reasonable with the long-range resistive-wall field. In fact, S. Heifets pointed out that unless one takes into account the diffusion time of the field, the multi-turn summation diverges [3]. Secondly the single turn effect is much stronger in the horizontal plane than the vertical due to the machine optics. These two results suggest that the diffusion time, which is introduced in Ref. 3, is much longer in the vertical plane than the horizontal, to explain the comparable tune shifts. The number of turns needed to reproduce the measured tune shifts is nearly 40 turns horizontally and 600 turns vertically (Fig. 4). On the other hand, as may be anticipated, the calculated tune shifts between head and tail in a bunch train (e.g. 1/3 filling) are nearly free of the multi-turn effect (Fig. 5).
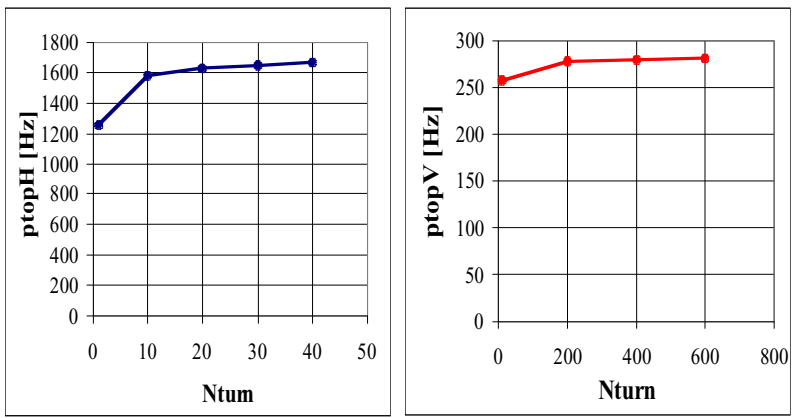

Figure 5: Calculated dependence of head-to-tail betatron tune shift on multi-turns.

Head to tail tune shifts were experimentally measured (Fig. 6). Both the horizontal and vertical tune shifts were in good agreement with those expected. In particular, we found $\Delta Q_{H} \gg \Delta Q_{V}$ (Fig. 7).

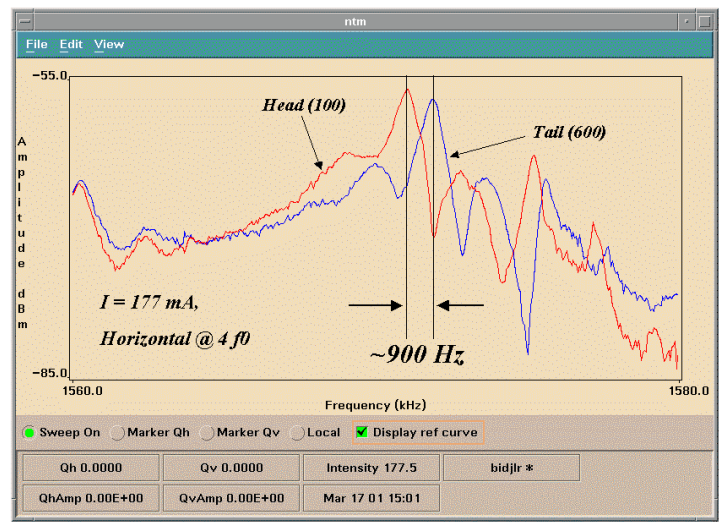

Figure 6: Measured horizontal tune shift between the head and the tail of a bunch train in $1 / 3$ filling.

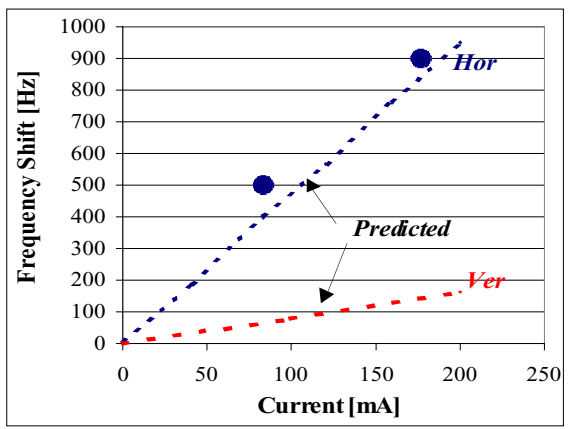

Figure 7: Comparison of head-to-tail tune shift between the calculated and the measured. 
An attempt was made to pursue the source of the tune shifts by taking the difference in the betatron phase advance, measured with a turn-by-turn BPMs, between 5 and $200 \mathrm{~mA}$ in $1 / 3$ filling. A continuous increase in the difference found is in line with the hypothesis that the low-gap chambers distributed around are the source [4].

\section{IMPACT ON SINGLE BUNCH}

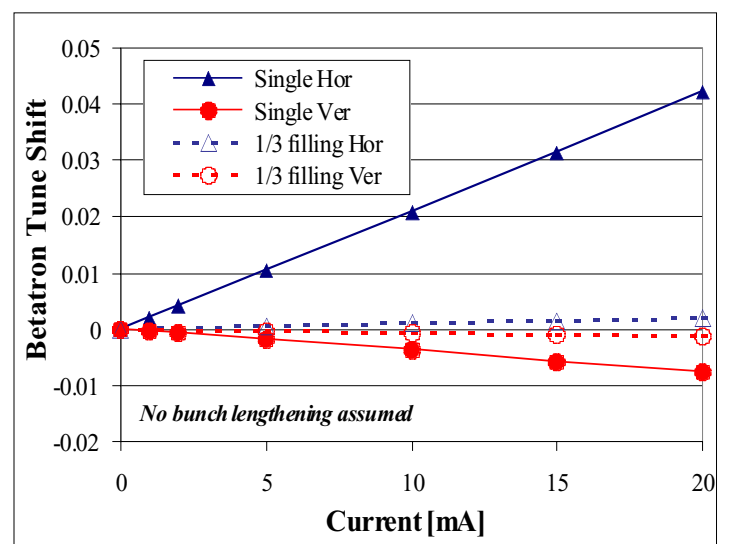

Figure 8: Comparison of calculated betatron tune shifts between single and multibunch (1/3 filling).

In the developed code, a "self force" was added to compute the intra bunch effect. The wake force was averaged over the particle distribution in a bunch to deduce an effective focusing strength felt by a single bunch. Due to the strong short-range component of the resistive-wall wake field, a significant detuning in single bunch was found. On the other hand, the multi-turn effect was expectedly negligible. Again due to the optics, tune shifts are enhanced in the horizontal plane (Fig. 8).

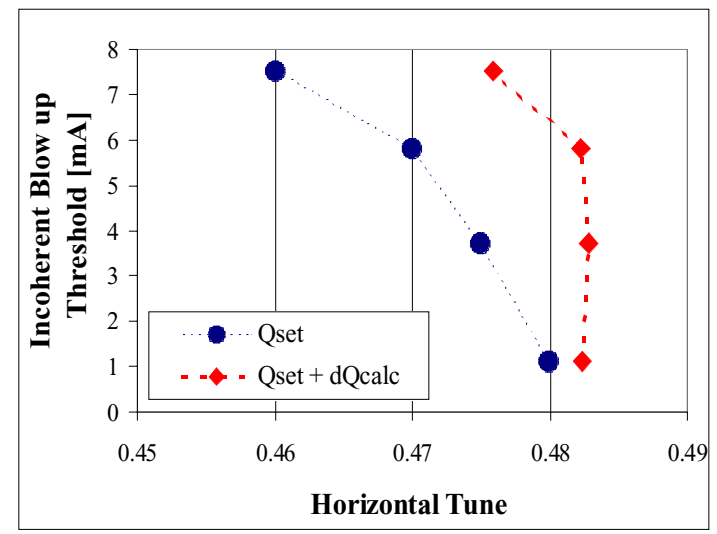

Figure 9: Measured incoherent blow-up threshold in single bunch as a function of the zero-current tune.

The obtained numerical finding goes well with some of the observations: 1) A high sensitivity of a high current single bunch to the horizontal half-integer resonance, which may blow up the beam incoherently and saturate injection. 2) Horizontal coherent mode frequency shifts [5]. At zero chromaticity, the mode 0 is only slightly detuned, while other head-tail modes are focused by a similar degree. It may be that the mode zero is under the influence of counteracting inductive impedance and a mean resistive-wall field, while others are only focused by the latter. In fact, the observed detuning of modes could not be fitted with a simple BBR impedance.

The horizontal incoherent blow up due to the halfinteger resonance was followed for different zero current tunes. The large variation observed on the threshold current, and in particular, the constancy of the expected tune at the threshold, are in favour of the calculated incoherent tune shift (Fig. 9).

\section{CONCLUSION}

The incoherent tune shifts deduced from the computed resistive-wall wake fields led us to assume that the diffusion time of the field is much longer vertically than horizontally, to explain the observed horizontal and vertical tune shifts, which are comparable. Whereas, calculated tune shifts between the head and tail in a bunch train were nearly free of the multi-turn effect, and were found to agree well with the measurement, including the fact that vertical shifts are much smaller than horizontal ones. The numerical analysis furthermore showed that there is a significant tune shift with current in single bunch due to the strong short-range resistive-wall wake field. Some experimental evidence in favour of this finding has been obtained. The points to be subsequently investigated are as follows: Any possible contribution to the tune shift from the machine heating. The effect of 50 $\mu \mathrm{m}$ copper coating on the narrowest gap chambers, which was assumed to be small. Justification of the large asymmetry in the diffusion time between horizontal and vertical. Possible link of the concerned tune shift to the observed extra stability in non-continuous beam fillings (via Landau damping).

\section{ACKNOWLEGDGEMENT}

The author is especially grateful to Jean-Luc Revol for his contribution to the measurement of head to tail tune shifts and for discussions. He thanks L. Farvacque, J. Jacob, E. Plouviez, K. Scheidt and A. Ropert for their general support on this work.

\section{REFERENCES}

[1] R.L. Gluckstern, J. van Zeijts, B. Zotter, "Coupling Impedance of Beam Pipes of General Cross Section", CERN SL/AP 92-25, Geneva, June 1992.

[2] K. Yokoya, "Resistive Wall Impedance of Beam Pipes of General Cross Section", Part. Acc., 41 (1993) 221.

[3] S. Heifets, "Tune Shift due to Asymmetry of the Resistive Beam Pipe", SLAC-PUB-7985, Stanford, October 1998

[4] R. Nagaoka, L. Farvacque, "Analysis of Normal and Skew Quadrupole Errors at the ESRF", this conference.

[5] J.L. Revol, R. Nagaoka, "Observation, Modelling and Cure of Transverse Instabilities at the ESRF", this conference. 